\title{
Metodología multicriterio para la selección de proveedores bajo consideraciones de riesgo
}

\author{
Multi-Criteria methodology for the selection of suppliers under risk considerations
}

\author{
C. A. Parra-Calderón iD; J. C. Osorio-Gómez iD ; J.C. Escandón-López iD
}

\begin{abstract}
One of the aspects that has become more relevant in the supply chains management is the one related to the supplier management. In this process, the selection is a fundamental activity, since depending of the accuracy of this decision will be derived the results of the productive system. It has been demonstrated in the literature that supplier selection is a multicriteria problem and therefore, requires the use of appropriate methodologies for this type of problem. One of the multi-criteria methodologies that is gaining strength in the field of multi-criteria decision-making problems is the diffuse AHP (Fuzzy Hierarchic Analytic Process), which combines the traditional AHP with fuzzy logic. Another key aspect in the management of modern supply chains is the one related to risks. Managing risks properly is vital for the organizations survival. This article proposes an application of the fuzzy AHP technique for the supplier selection including the operational risk within the selection criteria. The proposed methodology is applied in the selection process of a raw material supplier of a manufacturing company in Colombia, yielding satisfactory results for the organization when selecting the supplier that responds in the best way to the set of criteria established by the company. The proposed methodology represents an effective method to evaluate different suppliers according to a set of criteria selected by a group of decision makers through the use of different comparison matrices.
\end{abstract}

Index Terms - Fuzzy AHP, fuzzy logic, operational risk, supplier selection, multi-criteria techniques.

Resumen- Uno de los aspectos que mayor relevancia viene cobrando en la gestión de las cadenas de suministro es el relacionado con la gestión de los proveedores. En este proceso, la selección es una actividad fundamental, puesto que de lo acertada que sea esta decisión derivarán los resultados del sistema productivo. Se ha demostrado en la literatura que la selección de proveedores es un problema multicriterio y por tanto, requiere la utilización de metodologías apropiadas para este tipo de problemas. Una de las metodologías multicriterio que viene tomando fuerza en el campo de los problemas de toma de decisión multicriterio es el AHP difuso (Proceso analítico jerárquico difuso), el cual combina el tradicional AHP con la lógica difusa. Otro aspecto fundamental dentro de la gestión de las cadenas de

Este manuscrito fue enviado el 11 de noviembre de 2018 y fue aceptado el 29 de marzo de 2019.

C. A. Parra-Calderón, Ingeniero Industrial egresado de la Universidad del Valle, Santiago de Cali, Colombia, vinculado a Idemia S.A desde 2018.

(email: cristian.parra@correounivalle.edu.co).

J. C. Osorio-Gómez, Ingeniero Industrial egresado de la Universidad del Valle, Santiago de Cali, Colombia, Doctor en ingeniería de la misma universidad y profesor titular de la Escuela de Ingeniería Industrial. suministro modernas es el relacionado con los riesgos. Gestionar adecuadamente los riesgos es vital para la supervivencia de las organizaciones. Este artículo propone una aplicación del AHP difuso para la selección de proveedores involucrando dentro de los criterios de selección el riesgo operacional. La metodología propuesta es aplicada en el proceso de selección de un proveedor de materia prima de una empresa manufacturera en Colombia, arrojando resultados satisfactorios para la organización al seleccionar el proveedor que responde de la mejor manera al conjunto de los criterios establecidos por la empresa. La metodología propuesta representa un método eficaz para evaluar diferentes proveedores de acuerdo a un conjunto de criterios seleccionados por un grupo de responsables en el proceso de toma de decisión mediante el uso de diferentes matrices de comparación.

Palabras claves - Fuzzy AHP, fuzzy logic, riesgo operacional, selección de proveedores, técnicas multicriterio.

\section{INTRODUCCIÓN}

$\mathrm{A}$ CTUALMENTE el mercado es altamente competitivo y cambiante, por ello la gestión de la cadena de suministro juega un papel fundamental para las organizaciones que buscan permanecer en el mismo. La selección de proveedores es una decisión que hace parte de la gestión de la cadena de suministro y es considerada como uno de los problemas clave que enfrentan las organizaciones en busca de garantizar el correcto desarrollo de sus operaciones y mantener la competitividad en el mercado.

En la búsqueda de mejores desempeños competitivos, una tendencia mundial es la gestión de las cadenas de abastecimiento. Para este propósito, la selección de proveedores constituye una decisión estratégica de alto impacto en el desempeño de la organización. [1]. Dentro de la gestión de la cadena de suministro la selección de proveedores es considerada como una de las decisiones más difíciles y trascendentes para las organizaciones, ya que ésta determina en gran medida el éxito o fracaso de las mismas.

(email: juan.osorio@correounivalle.edu.co).

J.C. Escandón-López, Ingeniero Industrial egresado de la Universidad del Valle, Santiago de Cali, Colombia, actualmente estudiante de Maestría en Administración de Universidad del Valle, Santiago de Cali, Colombia, vinculado al Grupo Coomeva desde 2018. (email: juan.c.escandon@correounivalle.edu.co). 
A medida que pasa el tiempo las organizaciones tienden a depender más de sus proveedores, es por ello que el proceso de la selección de proveedores ha sido ampliamente estudiado y se han desarrollado múltiples técnicas, que consideran criterios tanto cuantitativos como cualitativos para garantizar así una correcta selección de los mismos, acorde a las necesidades de la organización.

El análisis de la literatura realizado indicó que la calidad, el precio y el tiempo de entrega son criterios claves que frecuentemente se consideran en el proceso de selección de proveedores. Sin embargo, seleccionar a los proveedores adecuados implica mucho más que evaluar factores como esos, se deben de tener en cuenta también un amplio rango de criterios tanto cuantitativos como cualitativos, entre los cuales se destacan:

- Servicio al cliente.

- Capacidad de producción.

- Administración.

- Confianza.

- Flexibilidad operativa.

- Seguridad.

- Reputación.

Además de considerar criterios como los mencionados anteriormente, el problema de selección de proveedores también debe de considerar diferentes criterios asociados al riesgo en la cadena de suministro. Los riesgos podrían deberse a acciones originadas por el hombre o a eventos naturales. [2]. O tal como se establece en: [3][4]. se puede hablar de los riesgos de la cadena de suministro como interno y externo, así como por el nivel de capacidad de control.

Los riesgos en las cadenas de suministro son dinámicos en naturaleza, frecuencia y severidad. Algunos ejemplos de riesgo en la cadena de suministro, y que pueden ser considerados en el proceso de selección de un proveedor son:

- Riesgo de suministro: Asociado con la disponibilidad del suministro por parte de los proveedores, esto afecta la capacidad de la empresa para satisfacer la demanda del cliente.

- Variabilidad de la demanda: Asociado a la capacidad del proveedor para suministrar materias primas e insumos en épocas de alta demanda.

- Variabilidad en los tiempos de procesamiento del proveedor: Esto puede afectar los tiempos de entrega de las materias primas e insumos.

- Riesgo cambiario: En caso de que el proveedor sea extranjero existe riesgo en el momento de efectuar el pago, debido a la variación de la TRM.

- Desastres naturales: Riesgo asociado a la ocurrencia de desastres naturales que puedan afectar la producción, comunicación y entrega de las materias primas e insumos por parte del proveedor.

Los riesgos asociados a la cadena de suministro son numerosos y complejos por las diferentes implicaciones de los mismos sobre las operaciones de las organizaciones, es por ello que en la presente metodología solo se consideran los riesgos de carácter operacional, entendiéndose como riesgos operacionales aquellos asociados a las personas, procesos $\mathrm{y}$ equipos.

Los procesos de selección de proveedores adquieren complejidad por el número de alternativas y criterios asociados a las mismas, es por ello que es necesario contar con técnicas que permitan discernir sobre las alternativas, de manera que sea considerado el efecto de los múltiples criterios, y la solución responda a todos ellos de manera consolidada (global) y no individual (parcial). [5]. Muchos de estos criterios y alternativas varían de acuerdo a los objetivos estratégicos de la organización, y a la experiencia del tomador de decisiones, además algunas veces estos criterios y alternativas entran en conflicto entre sí; por esta razón el problema de la selección de proveedores es considerado como un problema multicriterio, en el cual se deben emplear técnicas pertinentes para abordarlo que permitan identificar aquellos proveedores que cuenten con el más alto potencial de satisfacer las necesidades de la organización.

Existen muchos métodos para tratar el problema de la selección de proveedores incluyendo el proceso analítico jerárquico (AHP), los procesos de red analítica (ANP), análisis envolvente de datos (DEA), teoría de conjuntos difusos (FST), algoritmos genéticos (GA), programación por metas (GP), técnica de calificación de atributos múltiples sencillos (SMART) y otros métodos. [6]. Los cuales permiten disminuir la subjetividad humana implicada en el proceso de selección, garantizando así la elección del mejor proveedor que se ajuste a los criterios requeridos por la organización, y que represente una solución al problema de la incertidumbre, asociada a realizar una elección errada.

Considerando los aspectos citados anteriormente, y dada la complejidad del problema y las consecuencias qué este puede causar al interior de las organizaciones, es necesario desarrollar una metodología qué permita realizar una efectiva selección de proveedores y que disminuya la incertidumbre asociada en el proceso.

\section{Medotodología}

Se ha seleccionado el proceso analítico jerárquico difuso (Fuzzy $A H P$ ), por su amplia aceptación y aplicación en los diferentes problemas que involucran la toma de decisiones con múltiples criterios. Además, porque al incorporar la lógica difusa, permite incorporar la incertidumbre asociada a los juicios de los tomadores de decisiones para garantizar la selección del mejor proveedor con base en los criterios considerados por la organización.

La figura 1 describe los pasos de la metodología propuesta a través de un flujograma, el método de AHP difuso usado en el desarrollo de la metodología se basó en: [7]. A continuación, se describen cada uno de los pasos. 


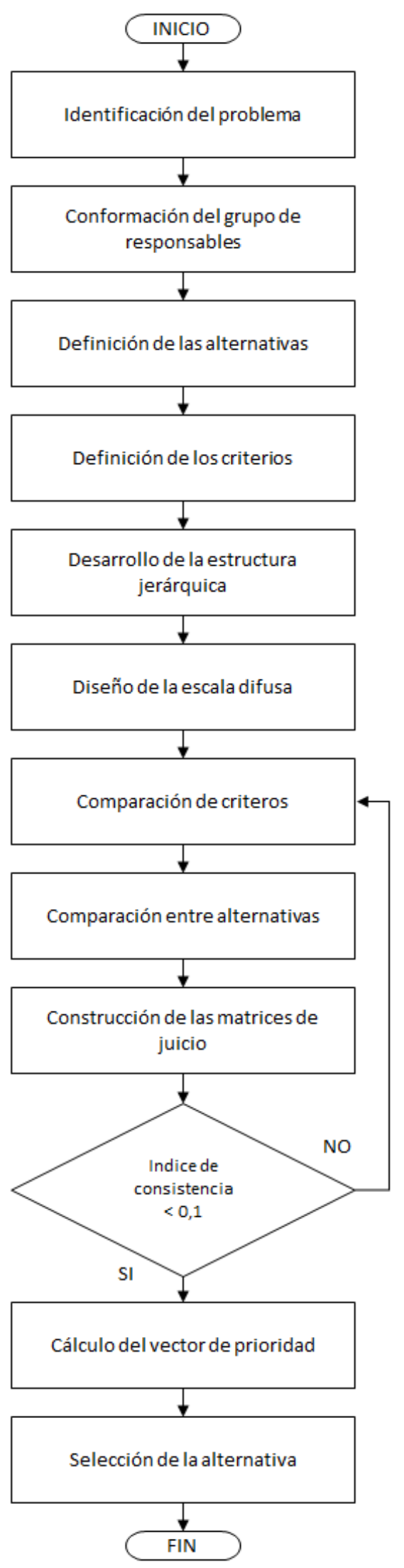

Fig 1. Flujograma: Metodología Propuesta. [8].

\section{A. Identificación del problema}

Este paso depende de cada caso de aplicación en particular, se deben de identificar la materia prima, el insumo o servicio que se desea adquirir y las alternativas de proveedores que suministran dichos productos, también se deben de identificar los problemas asociados a la no satisfacción de los criterios establecidos por la organización para el insumo, materia prima o producto deseado producto de la red de proveedores actuales.

\section{B. Conformación del grupo de responsables}

Una vez identificado el problema de selección de proveedores se debe de conformar un grupo interdisciplinario con las personas involucradas en el proceso productivo en el que se utilice la materia prima, insumo o servicio que se desea adquirir, también se debe incluir en este grupo de personas a aquellas encargadas de tomar las decisiones en el proceso de selección de proveedores.

\section{Definición de los criterios}

Cada caso de aplicación en particular cuenta con unas condiciones específicas, los criterios de selección del proveedor deben ser seleccionados por un consenso entre el grupo de responsables y deben estar alineados con los objetivos y necesidades de la organización.

\section{Desarrollo de la estructura jerárquica para los criterios y las alternativas}

Con base en: [7]. para aplicar AHP difuso (FAHP), los criterios de evaluación y las alternativas deben ser estructurados en diferentes niveles de jerarquía. Para esto se debe construir un esquema de árbol que resuma las interrelaciones entre los criterios y las alternativas; en la parte superior del esquema debe figurar siempre el objetivo principal o la meta, en los niveles inferiores deben figurar el conjunto de criterios y finalmente en la base del árbol deberán figurar las alternativas (ver Figura 2). No existe restricción respecto a la cantidad de niveles ni al número de elementos de cada nivel. 


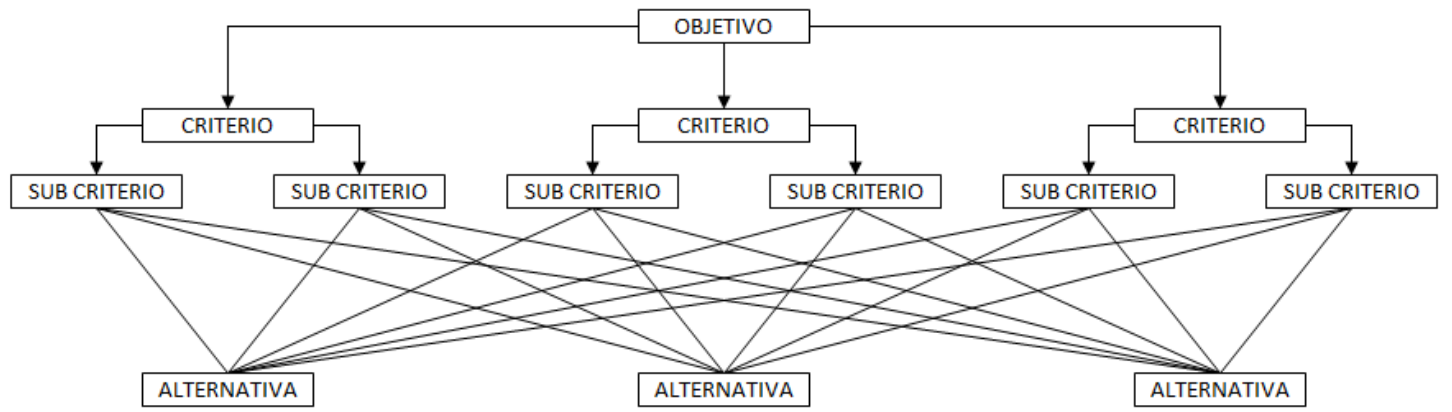

Fig 2. Estructura FAHP. Adaptado de. [9].

\section{E. Representación difusa de los juicios de comparación}

Una vez construida la jerarquía se debe de realizar la conversión de la escala de calificación de 9 puntos del AHP tradicional propuesta por: [10][11][12]. a una escala de números triangulares difusos, con el objetivo de introducir los juicios lingǘśsticos emitidos por los responsables del proceso de selección obtenidos en las diferentes entrevistas y encuestas e introducirlos en el modelo propuesto.

Los números triangulares T1, T3, T5, T7 y T9 son usados para representar los juicios desde igual hasta extremadamente preferido o importante, mientras que $\mathrm{T} 2, \mathrm{~T} 4, \mathrm{~T} 6$ y $\mathrm{T} 8$ representan los valores intermedios. La escala difusa propuesta para la metodología se puede apreciar en la Tabla I.

TABLA I.

EsCala Difusa De CALIFICACión PROPUESTA.

\begin{tabular}{|c|c|c|c|}
\hline $\begin{array}{c}\text { Notación } \\
\text { del } \\
\text { número } \\
\text { difuso }\end{array}$ & $\begin{array}{c}\text { Número } \\
\text { asociado } \\
\text { en la } \\
\text { escala de } \\
\text { saaty } \\
\end{array}$ & $\begin{array}{c}\text { Escala } \\
\text { triangular } \\
\text { difusa } \\
\text { propuesta }\end{array}$ & $\begin{array}{c}\text { Interpretación } \\
\text { verbal de la escala }\end{array}$ \\
\hline $\mathrm{T} 1$ & 1 & $(1,1,2)$ & $\begin{array}{l}\text { Igual importancia de } \\
\text { ambos elementos. } \\
\text { Moderada }\end{array}$ \\
\hline $\mathrm{T} 3$ & 3 & $(2,3,4)$ & $\begin{array}{l}\text { importancia de un } \\
\text { elemento sobre otro. }\end{array}$ \\
\hline $\mathrm{T} 5$ & 5 & $(4,5,6)$ & $\begin{array}{l}\text { un elemento sobre } \\
\text { otro. }\end{array}$ \\
\hline $\mathrm{T} 7$ & 7 & $(6,7,8)$ & $\begin{array}{l}\text { Muy fuerte } \\
\text { importancia de un } \\
\text { elemento sobre otro. }\end{array}$ \\
\hline T9 & 9 & $(8,9,9)$ & $\begin{array}{l}\text { Extrema importancia } \\
\text { de un elemento sobre } \\
\text { otro. }\end{array}$ \\
\hline $\begin{array}{l}\text { T2, T4, } \\
\text { T6, T8 }\end{array}$ & $2,4,6,8$ & $\begin{array}{l}(1,2,3), \\
(3,4,5), \\
(5,6,7), \\
(7,8,9)\end{array}$ & Juicios intermedios. \\
\hline
\end{tabular}

\section{F. Construcción de las matrices de juicio difusas para FAHP}

Con base en la jerarquía, los juicios lingüísticos del grupo de responsables y la escala construida en los pasos anteriores, se procede a la construcción de las matrices de juicio con el fin de comparar los elementos en los niveles dados para así estimar sus importancias relativas en relación con el elemento del nivel superior.

Estas matrices se construyen con base en la escala de calificación propuesta en la Tabla 1. y en las calificaciones lingüísticas dadas por el grupo de responsables para el caso de aplicación en el que se desee implementar la metodología. La media geométrica se aplica para integrar los pesos difusos adjudicados por los tomadores de decisiones. La media geométrica para números triangulares difusos se calcula a partir de la siguiente ecuación. [14]:

$$
\widehat{r}_{l}=\left(\prod_{k=1}^{n} \widehat{d_{l k}}\right)^{\frac{1}{n}}
$$

\section{G. Matriz de juicio nivel 1 (Criterios)}

En la matriz de comparación de criterios se usan los números triangulares de la escala propuesta en la Tabla 1. para expresar las preferencias entre los diferentes criterios con respecto a la meta, de estas preferencias se obtiene la primera matriz de comparación por pares entre los criterios con respecto al objetivo. En la Tabla II se presenta la estructura de comparación de criterios.

TABLA II.

MATRIZ DE JUICIO De Los CRITERIOS. ADAPTADO DE. [13].

\begin{tabular}{|c|c|c|c|c|}
\hline Objetivo & Criterio 1 & Criterio 2 & $\ldots$ & Criterio n \\
\hline $\begin{array}{l}\text { Criterio } \\
1\end{array}$ & $(1,1,1)$ & $\begin{array}{l}\text { Comparación } \\
\text { entre los } \\
\text { criterios } 1 \text { y } \\
2 .\end{array}$ & $\cdots$ & $\begin{array}{l}\text { Comparación } \\
\text { entre los } \\
\text { criterios } 1 \quad y \\
\text { n. }\end{array}$ \\
\hline $\begin{array}{l}\text { Criterio } \\
2\end{array}$ & $\begin{array}{l}\text { Inverso de la } \\
\text { comparación } \\
\text { entre los } \\
\text { criterios } 1 \text { y } \\
2 .\end{array}$ & $(1,1,1)$ & $\ldots$ & $\begin{array}{l}\text { Comparación } \\
\text { entre los } \\
\text { criterios } 2 \text { y } \\
\text { n. }\end{array}$ \\
\hline$\cdots$ & $\ldots$ & $\ldots$ & $(1,1,1)$ & $\begin{array}{l}\text { Comparación } \\
\text { entre los } \\
\text { criterios } n y \\
\text { n. }\end{array}$ \\
\hline $\begin{array}{l}\text { Criterio } \\
\text { n }\end{array}$ & $\begin{array}{l}\text { Inverso de la } \\
\text { comparación } \\
\text { entre los } \\
\text { criterios } 1 \text { y } \\
\text { n. }\end{array}$ & $\begin{array}{l}\text { Inverso de la } \\
\text { comparación } \\
\text { entre los } \\
\text { criterios } 2 \text { y } \\
\text { n. }\end{array}$ & $\begin{array}{l}\text { Inverso de la } \\
\text { comparación } \\
\text { entre los } \\
\text { criterios n y } \\
\text { n. }\end{array}$ & $(1,1,1)$ \\
\hline
\end{tabular}


De acuerdo a la escala difusa de calificación propuesta en la tabla 1. por ejemplo, si el tomador de decisiones evalúa el criterio 1 (C1) y determina que este es moderadamente importante sobre el criterio 2 (C2), entonces este juicio tomará la escala de calificación difusa $(2,3,4)$; por otro lado, cuando se compare al criterio 2 (C2) con respecto al criterio $1(\mathrm{C} 1)$, este juicio tomará la escala triangular difusa $(1 / 4,1 / 3,1 / 2)$, el cual corresponde a su inverso de acuerdo a la metodología del AHP. Con base en la matriz de juicio nivel 1 (criterios) se construyen las matrices de nivel 2, que siguen la misma estructura de la matriz de juicio nivel 1.

\section{H. Matriz de juicio nivel 2, de las alternativas}

Se usa la escala lingüística de números triangulares difusos para expresar las preferencias entre las diferentes alternativas con respecto al criterio 1 , de estas preferencias se obtiene la primera matriz de comparación por pares entre las alternativas con respecto al criterio 1. En la Tabla III. Se presenta la estructura de comparación de alternativas (proveedores).

TABLA III.

Matriz De Juicio De Los Proveedores. ADAPTADO DE. [13].

\begin{tabular}{|c|c|c|c|c|}
\hline Criterio 1 & Proveedor 1 & Proveedor 2 & $\ldots$ & Proveedor $n$ \\
\hline $\begin{array}{l}\text { Proveedor } \\
1\end{array}$ & $(1,1,1)$ & $\begin{array}{l}\text { Comparación } \\
\text { entre los } \\
\text { proveedores } \\
1 \text { y } 2 .\end{array}$ & $\cdots$ & $\begin{array}{l}\text { Comparación } \\
\text { entre los } \\
\text { proveedores } \\
1 \text { y n. }\end{array}$ \\
\hline $\begin{array}{l}\text { Proveedor } \\
2\end{array}$ & $\begin{array}{l}\text { Inverso de la } \\
\text { comparación } \\
\text { entre los } \\
\text { proveedores } \\
1 \text { y } 2 .\end{array}$ & $(1,1,1)$ & $\ldots$ & $\begin{array}{l}\text { Comparación } \\
\text { entre los } \\
\text { proveedores } \\
2 \text { y n. }\end{array}$ \\
\hline$\cdots$ & $\ldots$ & $\ldots$ & $(1,1,1)$ & $\begin{array}{l}\text { Comparación } \\
\text { entre los } \\
\text { proveedores } \\
\text { N y n. }\end{array}$ \\
\hline $\begin{array}{l}\text { Proveedor } \\
\text { n }\end{array}$ & $\begin{array}{l}\text { Inverso de la } \\
\text { comparación } \\
\text { entre los } \\
\text { proveedores } \\
1 \text { y n. }\end{array}$ & $\begin{array}{l}\text { Inverso de la } \\
\text { comparación } \\
\text { entre los } \\
\text { proveedores } \\
2 \mathrm{y} \mathrm{n} \text {. }\end{array}$ & $\begin{array}{l}\text { Inverso de la } \\
\text { comparación } \\
\text { entre los } \\
\text { proveedores } \\
\text { N y n. }\end{array}$ & $(1,1,1)$ \\
\hline
\end{tabular}

\section{Cálculo del índice de consistencia}

La técnica FAHP emplea un índice de consistencia que tiene como propósito medir cualquier inconsistencia tanto en los juicios de cada matriz de comparación como en toda la jerarquía. Para calcular el índice de consistencia se deben convertir las matrices de comparación difusas en matrices de números reales, las cuales serán usadas en el cálculo de la consistencia. Según. [15]. Un número triangular difuso denotado como $\mathrm{T}=(\mathrm{l}, \mathrm{m}, \mathrm{u})$ será convertido en un número real de la siguiente manera:

$$
M_{\text {real }}=\frac{(4 m+l+u)}{6}
$$

Teniendo la matriz de número reales y el vector de prioridad obtenido en el paso anterior, el proceso es el mismo que el del índice de consistencia en el AHP tradicional tal como se realiza en el trabajo de: [10][11][12].

\section{J. Cálculo del vector de prioridad}

El vector de prioridad se calcula para cada una de las matrices de juicio en todos los niveles de complejidad, este vector define la prioridad que tiene cada uno de los elementos de la matriz sobre los demás, para realizar el cálculo del vector de prioridad en FAHP se requiere primero realizar dos cálculos iniciales, los cuales serán explicados a continuación:

\section{K. Cálculo de los valores de comparación difusos}

Los valores de comparación difusos son un consolidado de cada uno de los números de la escala de comparación difusa que se usaron para comparar cada uno de los elementos de la matriz con los demás elementos, se calculan a partir de la media geométrica (tal como se realiza para integrar los juicios de los tomadores de decisiones). A continuación, se presenta un ejemplo en la tabla IV.

TABLA IV.

EJEMPLo De VALORES De COMPARACIÓN Difusos

\begin{tabular}{|c|c|c|c|c|c|}
\hline & Criterio 1 & Criterio 2 & Criterio 3 & Criterio 4 & Criterio 5 \\
\hline $\begin{array}{l}\text { Criteri } \\
\text { o } 1\end{array}$ & $(1,1,1)$ & $(1,1,1)$ & $(4,5,6)$ & $(6,7,8)$ & $(4,5,6)$ \\
\hline $\begin{array}{l}\text { Criteri } \\
02\end{array}$ & $(1,1,1)$ & $(1,1,1)$ & $(4,5,6)$ & $(6,7,8)$ & $(6,7,8)$ \\
\hline $\begin{array}{l}\text { Criteri } \\
\text { o } 3\end{array}$ & $\begin{array}{l}(1 / 6,1 / 5,1 / \\
4)\end{array}$ & $\begin{array}{l}(1 / 6,1 / 5,1 / \\
4)\end{array}$ & $(1,1,1)$ & $\begin{array}{l}(1 / 4,1 / 3,1 / \\
2)\end{array}$ & $(2,3,4)$ \\
\hline $\begin{array}{l}\text { Criteri } \\
\text { o } 4\end{array}$ & $\begin{array}{l}(1 / 8,1 / 7,1 / \\
6)\end{array}$ & $\begin{array}{l}(1 / 8,1 / 7,1 / \\
6)\end{array}$ & $(2,3$ & $(1,1,1)$ & $\begin{array}{l}\text { (1/6,1/5,1/ } \\
4)\end{array}$ \\
\hline $\begin{array}{l}\text { Criteri } \\
\text { o } 5\end{array}$ & $\begin{array}{l}(1 / 6,1 / 5,1 / \\
4)\end{array}$ & $\begin{array}{l}(1 / 8,1 / 7,1 / \\
6)\end{array}$ & $\begin{array}{l}(1 / 4,1 / 3,1 / \\
2)\end{array}$ & $(4,5,6)$ & $(1,1,1)$ \\
\hline
\end{tabular}

$$
\begin{aligned}
\widehat{r}_{l}=\left(\prod_{k=1}^{n} \widehat{d_{l k}}\right)^{\frac{1}{n}} \text { (3) } \\
=\left[(1 * 1 * 4 * 6 * 4)^{\frac{1}{5}} ;(1 * 1 * 5 * 7 * 5)^{\frac{1}{5}} ;(1 * 1 * 6 * 8 * 6)^{\frac{1}{5}}\right] \\
=[2.49 ; 2.81 ; 3.10]
\end{aligned}
$$

Teniendo los valores de comparación para cada uno de los elementos de la matriz, se procede a realizar una suma de cada uno de los números triangulares de los valores de comparación. A este número triangular obtenido en la sumatoria se le calcula el inverso.

\section{Cálculo de los pesos difusos relativos}

A cada uno de los elementos de la matriz se le realiza el cálculo de sus pesos difusos relativos, multiplicando su respectivo valor de comparación difuso por el inverso del total obtenido, de la siguiente manera:

$$
\widehat{w_{l}}=\widehat{r}_{l} *\left(\widehat{r}_{1}+\widehat{r}_{2}+\cdots+\widehat{r}_{n}\right)^{-1}=\left(l w_{l}, m w_{l}, u w_{l}\right)
$$

A continuación, se presenta un ejemplo para el cálculo en la Tabla V.

TABLA V. EJEMPLO PESOS Difusos Relativos

\begin{tabular}{llll}
\hline Criterios & RI & & \\
\hline Criterio 1 & 2,49 & 2,81 & 3,1 \\
Criterio 2 & 2,7 & 3 & 3,29 \\
Criterio 3 & 0,43 & 0,53 & 0,66 \\
Criterio 4 & 0,35 & 0,41 & 0,49 \\
Criterio 5 & 0,46 & 0,54 & 0,66 \\
Total & 6,43 & 7,3 & 8,2 \\
Inverso & 0,16 & 0,14 & 0,12 \\
\hline
\end{tabular}




$$
\begin{gathered}
\frac{\text { Invertido }}{0,12} 0,14 \quad 0,16 \\
\widehat{w}_{1}=[(2.49 * 0.12) ;(2.81 * 0.14) ;(3.10 * 0.16)] \\
=[0.304 ; 0.385 ; 0.483]
\end{gathered}
$$

Finalmente, el vector de prioridad será igual a los valores normalizados del promedio de los términos de cada uno de los pesos difusos relativos para cada elemento de la matriz de juicio, de la siguiente forma:

Promedio de cada peso difuso relativo:

$$
M_{l}=\frac{l w_{l}+m w_{l}+u w_{l}}{3}
$$

Normalización de los promedios:

$$
N_{l}=\frac{M_{l}}{\sum_{l=1}^{n} M_{l}}
$$

\section{Elección final de la alternativa}

Después de haber obtenido los vectores de prioridad a partir de matrices de juicio consistentes, se procede a multiplicar cada uno de los vectores de prioridad de las matrices en los niveles de juicio más bajos por los vectores de prioridad de la matriz en un nivel de juicio inmediatamente superior, y así sucesivamente hasta llegar a la cima de los niveles de juicio, donde se elegirá la alternativa con el peso más alto, puesto que esta será la mejor alternativa de acuerdo a los criterios y calificaciones propuestas en el proceso de toma de decisión.

\section{RESUltados}

En el presente artículo se desarrolló una metodología que combina el AHP con la lógica difusa para seleccionar a un proveedor de materia prima en una empresa manufacturera. La materia prima evaluada en el proceso de selección es una de las más importantes en la organización, puesto que es la más usada en los diferentes procesos y de la misma depende en gran medida la calidad de los productos que se producen.

A. Identificación del problema

La empresa cuenta actualmente con 130 referencias de un tipo de materia prima que es usada en diferentes procesos de producción.

Esta situación ha causado principalmente dos problemas al interior de la compañía:

- En inventario se cuenta con referencias de 3 proveedores distintos, lo cual causa tiempos perdidos debido al proceso de búsqueda de las referencias en el almacén.

- Debido al hecho de considerar el precio como único criterio de selección se presentan problemas de calidad en el producto final por causa de factores técnicos de la materia prima.

Estas situaciones han originado la necesidad en la empresa de contar únicamente con un proveedor de la materia prima estudiada, para que de esta forma la organización pueda entablar una relación de intercambio de información que permita la realización de planes de acción en pro de asegurar la calidad del producto y otros factores a un precio competitivo.
B. Conformación del grupo de responsables

El segundo paso corresponde a la conformación del grupo de responsables de tomar la decisión de selección de proveedores en la organización. En particular, se conformó un grupo de responsables con las siguientes personas, las cuales están relacionadas tanto en el proceso de selección de los proveedores como también en los procesos productivos en los cuales es usada la materia prima en cuestión:

- Gerente de planta.

- Superintendente de planta.

- Jefe de producción.

- Ingeniero de procesos.

- Jefe de laboratorio.

- Jefe de almacén.

- Director de compras e importaciones.

Una vez conformado el grupo de responsables para el proceso de selección, se realizaron una serie de entrevistas a los mismos, las cuales tenían como objetivo identificar aspectos claves del proceso de selección, como las alternativas o proveedores actuales de la materia prima analizada y los criterios de selección considerados por la organización para la selección de la misma.

\section{Definición de alternativas}

De acuerdo a la información recopilada en la entrevista con los participantes del proceso de selección de proveedores en la organización, se identificó que la materia prima analizada era comprada a 3 proveedores diferentes, los cuales por políticas de privacidad de la organización serán considerados como Proveedor 1, Proveedor 2 y Proveedor 3.

D. Definición de criterios

Se definieron los siguientes, incluyéndose tal como se esperaba, dos criterios relacionados con el riesgo operacional:

- Precio.

- Tiempo de entrega.

- Calidad.

- Capacidad de producción.

- Servicio al cliente.

- Riesgo de suministro.

- Riesgo de calidad.

A. Elección final de la alternativa

Después de aplicar toda la metodología se obtuvieron los resultados presentados en la tabla VI:

TABLA VI

ELECCIÓn FinAL De La ALTERnATIVA

\begin{tabular}{llll}
\hline Elección final & Proveedor 1 & Proveedor 2 & Proveedor 3 \\
\hline Riesgo de calidad & 0,09 & 0,01 & 0,03 \\
Riesgo de suministro & 0,08 & 0,02 & 0,05 \\
Precio & 0,03 & 0,03 & 0,03 \\
Tiempo de entrega & 0,11 & 0,05 & 0,07 \\
Calidad & 0,07 & 0,03 & 0,02 \\
Capacidad de producción & 0,13 & 0,02 & 0,07 \\
Servicio al cliente & 0,02 & 0,02 & 0,01 \\
Total & $\mathbf{0 , 5 3}$ & $\mathbf{0 , 1 9}$ & $\mathbf{0 , 2 8}$ \\
\hline
\end{tabular}

De acuerdo a los resultados obtenidos se observa que la alternativa Proveedor 1 tiene el más alto puntaje global, por lo tanto, se concluye que es el mejor proveedor de acuerdo a los 
objetivos de la organización y a los criterios y calificaciones establecidas por los responsables del proceso de selección de proveedores. Adicionalmente, se observa una diferencia importante entre esta alternativa y las otras dos, por ello, no es recomendable en caso de no tomar la decisión de comprar al proveedor 1, considerar uno de los otros dos. En este caso particular y debido a la amplia diferencia entre la alternativa 1 y las otras dos, sería mejor considerar otras alternativas en el caso de no poder realizar el proceso de compra con el proveedor número uno.

\section{CONCLUSIONES}

La metodología propuesta representa un método eficaz para evaluar diferentes proveedores de acuerdo a un conjunto de criterios seleccionados por un grupo de responsables en el proceso de toma de decisión mediante el uso de diferentes matrices de comparación.

Contar con una metodología para seleccionar a los proveedores adecuados de acuerdo a una serie de criterios considerados es una gran ventaja en la actualidad, ya que permite a las organizaciones satisfacer las necesidades de sus clientes en cuanto a productos, bienes o servicios; lo cual les permite ser más competitivas y generar mayores utilidades en el mercado global.

El AHP difuso es una herramienta importante que permite tomar decisiones tan importantes como la selección de un proveedor, incorporando todos los criterios que a juicio de la organización son importantes, y considerando para la decisión todas las opiniones y juicios de los involucrados.

\section{REFERENCIAS}

[1] Sarache Castro, W. A., Castrillón Gómez, Ó. D., \& Ortiz Franco, L. F. (2009). Selección de proveedores: una aproximación al estado del arte. Cuadernos de Administración, 22(38).

[2] Ravindran, A. R., Bilsel, R. U., Wadhwa, V., \& Yang, T. (2010). Risk adjusted multicriteria supplier selection models with applications. International Journal of Production Research, 48(2), 405-424. DOI:10.1080/00207540903174940.

[3] Wu, D., \& Olson, D. L. (2008). Supply chain risk, simulation, and vendor selection. International Journal of Production Economics, 114(2), 646-655. DOI:10.1016/j.ijpe.2008.02.013.

[4] Wu, D. D., Zhang, Y., Wu, D., \& Olson, D. L. (2010). Fuzzy multi-objective programming for supplier selection and risk modeling: A possibility approach. European Journal of $\begin{array}{lll}\text { Operational } & \text { Research, 200(3), 774-787. }\end{array}$ DOI:10.1016/j.ejor.2009.01.026.

[5] Herrera Umaña, M. F., \& Osorio Gómez, J. C. (2006). MODELO PARA LA GESTIÓN DE PROVEEDORES UTILIZANDO AHP DIFUSO. (Spanish). Estudios Gerenciales, 69-88. DOI:10.1016/S0123-5923(13)70015-9

[6] Dahel, N. (2003). Vendor selection and order quantity allocation in volume discount environments. MCB UP Ltd.

[7] Ayhan, M. B. (2013). A Fuzzy AHP Approach for Supplier Selection Problem: A Case Study in a Gearmotor Company. Internation Journal of Managing Value and Supply Chains (IJMVSC), 4(3), 11-23.DOI:10.5121/ijmvsc.2013.4302.

[8] Escandón López, J. C. \& Parra Calderon, C. A. (2016). Metodología multicriterio para la selección de proveedores bajo consideraciones de riesgo. bibliotecadigital.univalle.edu.co/

[9] Deng, X., Hu, Y., Deng, Y., \& Mahadevan, S. (2014). Supplier selection using AHP methodology extended by D numbers.
Expert Systems with Applications, 41(1), 156-167. DOI:10.1016/j.eswa.2013.07.018

[10] Saaty, T. L. (1999). Fundamentals of the analytic network process. Proceedings of the ISAHP 1999, 1-14. DOI:10.1007/s11518-0060158-y.

[11] Saaty. (1980). How to make a decision: The Analytic Hierarchy Process, 48. Retrieved from http://ac.els-cdn.com.

[12] Saaty, T. L. (2012). The Analytic Hierarchy Process in Conflict Management. International Journal of Conflict Management (Vol. 1). DOI: $10.1007 / 0387-23081-5$

[13] Osorio Gómez, J. C., Herrera Umaña, M. F., \& Vinasco, M. A. (2008). Modelo para la evaluación del desempeño de los proveedores utilizando AHP. Ingeniería y Desarrollo, (23).

[14] Lee, A. H. I., Chen, W. C., \& Chang, C. J. (2008). A fuzzy AHP and BSC approach for evaluating performance of IT department in the manufacturing industry in Taiwan. Expert Systems with Applications, 34(1), 96-107. DOI: 10.1016/j.eswa.2006.08.022

[15] Facchinetti, G., Ricci, R. G., \& Muzzioli, S. (1998). Note on ranking fuzzy triangular numbers. International Journal of Intelligent Systems, 13(7), 613-622.

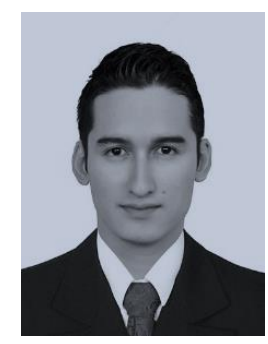

Cristian Andrés Parra Calderón, nacido el 27 de octubre de 1993 en Cali, Valle del cauca, Colombia. Ingeniero Industrial egresado de la Universidad del Valle, Santiago de Cali, Valle del Cauca, Colombia.

[16]Cristian Andres, trabajó en Sonoco Colombia durante los años 2015-2016 como estudiante en práctica y analista de seguridad industrial. Posteriormente estuvo vinculado a la empresa Postobón S.A. como supervisor de producción durante el año 2017. Actualmente se encuentra Vinculado a la empresa Idemia como planeador de producción.

ORCID: http://orcid.org/0000-0001-5872-312X

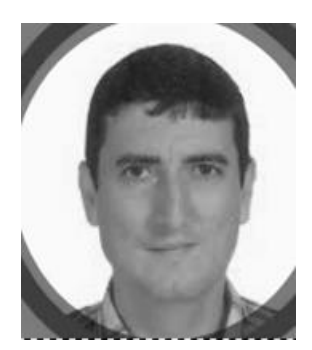

Juan Carlos Osorio Gómez, nacido el 20 de septiembre de 1975 en Buga, Valle del Cauca, Colombia. Ingeniero Industrial, Especialista en Logística, Magister en Ingeniería Industrial, Doctor en Ingeniería Industrial de la Universidad del Valle, Santiago de Cali, Valle del Cauca, Colombia.

Actualmente Profesor titular de la Escuela de Ingeniería Industrial de la Universidad del Valle.

ORCID: http://orcid.org/0000-0001-5625-5609

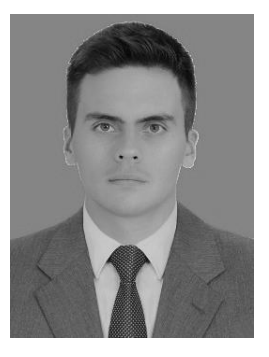

Juan Camilo Escandón López, nacido el 2 de febrero de 1995 en Palmira, Valle del Cauca, Colombia. Ingeniero Industrial egresado de la Universidad del Valle, Santiago de Cali, Valle del Cauca, Colombia, 2016. Actualmente estudiante de Maestría en Administración de la Universidad del Valle, Santiago de Cali, Valle del Cauca, Colombia. 
Juan Camilo, trabajó en Smurfit Kappa Colombia durante los años 2015 - 2016 cómo estudiante en práctica y programador de producción. Posteriormente estuvo vinculado a la Universidad del Valle cómo profesional contratista de la oficina de planeación y desarrollo institucional durante el año 2017, actualmente se encuentra vinculado a Coomeva

Emergencia Médica cómo Analista Nacional de Operaciones.

El Ing. Escandón se encuentra adscrito al Consejo Profesional Nacional de Ingeniería, bajo la matricula profesional $\mathrm{N}^{\circ}$ 76228-363600 VLL, expedida el 21 de julio del 2017.

ORCID: http://orcid.org/0000-0002-2396-6014 